\title{
ClpC acts as a negative regulator of competence in Streptococcus thermophilus
}

Correspondence

Leiv Sigve Håvarstein

sigve.havarstein@umb.no

Received 25 October 2010

Revised 1 March 2011

Accepted 24 March 2011

\section{Truls Johan Biørnstad and Leiv Sigve Håvarstein}

Department of Chemistry, Biotechnology and Food Science, Norwegian University of Life Sciences, N-1432 Ås, Norway

\begin{abstract}
The alternative sigma factor ComX is a key regulator of natural transformation in members of the genus Streptococcus. ComX controls expression of the late competence genes, which are essential for DNA binding, uptake and recombination. In Streptococcus pneumoniae, it has been demonstrated that ComX is degraded by ClpEP at the end of the competence period. In the present study we show that a different Clp protease complex, ClpCP, contributes to ComX degradation in Streptococcus thermophilus. Mutant strains lacking the ClpC chaperone displayed significantly increased transformability compared with the wild-type strain under conditions where ComX was expressed at relatively low levels. At higher expression levels, ClpCP appears to become saturated and unable to prevent the accumulation of ComX. Together, our results suggest that the role of $\mathrm{ClpC}$ is to mediate degradation of ComX when the sigma factor is produced in low amounts, i.e. when the environmental stimulus promoting competence development is weak. This would prevent $S$. thermophilus from developing the competent state at an inappropriate time and/or place.
\end{abstract}

\section{INTRODUCTION}

Recently, it was discovered that Streptococcus thermophilus, an economically important bacterium for the dairy industry, is competent for natural genetic transformation (Blomqvist et al., 2006b; Gardan et al., 2009; Fontaine et al., 2010). It was found that the alternative sigma factor ComX controls the core competence genes, i.e. all genes needed for DNA binding, uptake and recombination (Blomqvist et al., 2006b). This is in accordance with the situation in Streptococcus pneumoniae, where expression of the so-called late competence genes directly depends on ComX (Lee \& Morrison, 1999). In S. pneumoniae comX expression is regulated by a quorum-sensing-like system encoded by the comCDE genes (Håvarstein et al., 1995; Pestova et al., 1996). The system consists of a secreted competence-stimulating peptide (CSP) which is encoded by the $\operatorname{com} C$ gene, its membrane-anchored histidine kinase receptor ComD and the cognate response regulator ComE (for review, see Johnsborg \& Håvarstein, 2009). The genome of $S$. thermophilus contains a signal-transduction system of the ComCDE-type (BlpC and BlpRH) as well. However, this system does not control competence development, but regulates the production of several different bacteriocins and their immunity proteins (Fontaine et al., 2007). Regulation of competence development in $S$. thermophilus is based on a different quorum-sensing mechanism involving the oligopeptide pheromone ComS and the transcriptional activator ComR (Fontaine et al.,

Abbreviation: RLU, relative light units.
2010). Mature ComS is derived from the C-terminal end of a 24 aa ribosomally synthesized precursor. It is not known how ComS is processed and externalized, but the mature peptide ( $8 \mathrm{aa})$ is imported through the Ami oligopeptide transport system. Inside the cell mature ComS presumably binds to ComR, resulting in an active form of this transcription factor that drives transcription of the $\operatorname{com} X$ gene (Fontaine et al., 2010). The environmental cues or growth conditions triggering ComS production and spontaneous competence development in S. thermophilus have not been identified.

The evolutionarily conserved serine protease, ClpP, which monitors protein quality in general and controls the stability and activity of certain regulatory proteins, has been shown to act as a negative regulator of competence gene expression in S. pneumoniae (Chastanet et al., 2001; Robertson et al., 2002). The ClpP oligomer consists of 14 subunits arranged in two heptameric rings resembling a barrel. To gain proteolytic activity, the barrel-shaped proteolytic core must form a stable complex with hexameric rings of ATP-dependent chaperones termed Clp ATPases. It is the members of this protein family and not ClpP, that confer substrate specificity to the functional chaperone/ protease complex (Frees et al., 2007; Kress et al., 2009). In $S$. pneumoniae, ComX remains stable after competence induction in a mutant lacking $\mathrm{ClpP}$ and/or ClpE. This indicates that $\mathrm{ClpE}$ specifically recognizes ComX and associates with the ClpP proteolytic domain to degrade the alternative sigma factor (Sung \& Morrison, 2005; Piotrowski et al., 2009a). To investigate whether ComX is 
subjected to regulation by the Clp system in S. thermophilus LMG18311, we deleted several of the clp genes in this strain. Interestingly, we found that $\mathrm{ClpC}$-deficient strains displayed increased transformability compared with ClpCproficient strains under certain conditions. This result shows that $\mathrm{ClpC}$ acts as a negative regulator of competence in S. thermophilus.

\section{METHODS}

Bacterial strains and growth media. The following media were used to cultivate S. thermophilus LMG 18311 and mutants derived from this strain: Todd-Hewitt broth (TH) supplemented with $0.8 \%$ glucose (THG), Hogg-Jago glucose broth (HJG) consisting of $3 \%$ tryptone, $1 \%$ yeast extract, $0.2 \%$ beef extract, $0.5 \% \mathrm{KH}_{2} \mathrm{PO}_{4}$ and $0.5 \%$ glucose, HJG supplemented with $0.5 \%$ lactose (HJGL), HJGLS, which is HJGL supplemented with $0.4 \mathrm{M}$ D-sorbitol, and $\mathrm{C}$ medium (Lacks \& Hotchkiss, 1960). Agar plates were prepared by adding $1.5 \%$ $(\mathrm{w} / \mathrm{v})$ agar to the media. All incubations were carried out at $37{ }^{\circ} \mathrm{C}$ and all optical density measurements of bacterial cultures were performed spectrophotometrically at 492 or $550 \mathrm{~nm}$.

Electroporation of S. thermophilus LMG 18311. Cultures grown overnight at $37{ }^{\circ} \mathrm{C}$ were diluted 100 -fold in HJG prewarmed to $37{ }^{\circ} \mathrm{C}$ and incubated at this temperature until $\mathrm{OD}_{550}$ reached 0.3. Cultures (50 ml aliquots) were then diluted $1: 1$ in $\mathrm{HJG}\left(37^{\circ} \mathrm{C}\right)$ containing $20 \%$ glycine. After further incubation at $37^{\circ} \mathrm{C}$ for $1 \mathrm{~h}$, cells were harvested by centrifugation at $5000 \mathrm{~g}$ for $5 \mathrm{~min}$ at $4{ }^{\circ} \mathrm{C}$. Next the cells were washed with 1 vol ice-cold electroporation buffer $[5 \mathrm{mM}$ $\mathrm{KHPO}_{4}, 0.4 \mathrm{M}$ D-sorbitol, $10 \%$ (v/v) glycerol; pH 4.5] and pelleted by centrifugation at $5000 \mathrm{~g}$ for $10 \mathrm{~min}$ at $4{ }^{\circ} \mathrm{C}$. The washing step was repeated once. Finally, pelleted cells were resuspended in $2 \mathrm{ml}$ icecold electroporation buffer, divided into aliquots and frozen in liquid nitrogen. Electrocompetent cells were stored at $-80{ }^{\circ} \mathrm{C}$. Plasmid DNA electroporation was performed with a Bio-Rad MicroPulser unit as described previously (Blomqvist et al., 2006b).

Natural transformation of $S$. thermophilus LMG 18311. S. thermophilus LMG 18311 cells harbouring the pXL plasmid were grown overnight at $37{ }^{\circ} \mathrm{C}$ in THG supplemented with $2 \mu \mathrm{g}$ erythromycin $\mathrm{ml}^{-1}$. The next day the culture was diluted to $\mathrm{OD}_{550}$ 0.05 in the same medium prewarmed to $37{ }^{\circ} \mathrm{C}$ and $250 \mathrm{ng}$ peptide pheromone (STP) $\mathrm{ml}^{-1}$, which controls bacteriocin production in $S$. thermophilus LMG $18311\left(\mathrm{NH}_{2}\right.$-SGWMDYINGFLKGFGGQRTLPTKDYNIPQV-COOH) was immediately added. When $\mathrm{OD}_{550}$ reached 0.2 , culture samples $(1 \mathrm{ml})$ were transferred to Eppendorf tubes containing transforming DNA and further incubated for $2 \mathrm{~h}$ at $37^{\circ} \mathrm{C}$. Samples were then put on ice, serially diluted and spread on HJGL agar plates containing the appropriate antibiotic $(150 \mu \mathrm{g}$ spectinomycin $\mathrm{ml}^{-1}$ or $100 \mu \mathrm{g}$ kanamycin $\mathrm{ml}^{-1}$ ). An identical protocol was followed when competence in $S$. thermophilus was induced by the native pheromone, ComS ( $\mathrm{NH}_{2}$-LPYFAGCL-COOH), instead of STP. In this case $900 \mathrm{ng} C \mathrm{ComS} \mathrm{ml}{ }^{-1}$ was added to the culture. In the transformation experiments presented in Table $2,3 \mu \mathrm{g} \mathrm{ml} \mathrm{m}^{-1}$ of a plasmid (pCR2.1-TOPO) carrying a comEC gene disruption cassette (Blomqvist et al., 2006b) was used as transforming DNA. Curing of the pXL plasmid was performed by cultivating transformants in antibiotic-free medium for about 100 generations.

Construction of plasmids used for reporter assays and Western analysis. The construction of the plasmids used for the luciferase reporter assays, pXL, pEAP and pXP, has been described previously (Blomqvist et al., 2006b). To make the pXL-His plasmid we employed the comX_His_F and comX_His_R primers to amplify a fragment corresponding to the bacteriocin promoter and the adjacent $\operatorname{comX}$ gene. The pXL plasmid was used as template. To facilitate subsequent cloning, BamHI and EcoRV restriction sites were engineered into the 5 '-ends of comX_His_F and comX_His_R, respectively. In addition, a C-terminal $6 \times$ His-tag was inserted into the comX_His_R primer after the last amino acid of the ComX protein. The PCR fragment obtained with the comX_His_F and comX_His_R primers was first cloned into the pCR 2.1-TOPO vector (Invitrogen). Next the fragment was excised from this vector by cleavage with BamHI and EcoRV and ligated into the pEAP plasmid precleaved with $B a m \mathrm{HI}$ and SmaI. The resulting plasmid was designated pXL-His. All PCRs were carried out with the Phusion high-fidelity DNA polymerase (Finnzymes) and the final constructs were checked by complete sequencing.

Construction of mutants. The various target genes were disrupted by replacing the respective genes with a spectinomycin $(c l p C, c l p E$, $c l p L$ and $c l p X)$ or kanamycin $(c l p P$ and $\operatorname{com} X)$ resistance cassette. This was achieved by double-crossover homologous recombination using a PCR fragment consisting of the antibiotic resistance gene flanked by $\sim 1000 \mathrm{bp}$ DNA fragments corresponding to the flanking $5^{\prime}$ and $3^{\prime}$ regions of the target gene. In the first step the primer pairs Spec-F(A)/ Spec-R or Kana-F/Kana-R were used to amplify the spectinomycin or kanamycin resistance marker, respectively (Table 1). Plasmid pR412 was the source of the synthetic spectinomycin resistance cassette (Martin et al., 2000), while the kanamycin resistance gene was amplified from the pFW13 vector (Podbielski et al., 1996). Next, PCR fragments corresponding to the flanking regions of the target genes were amplified by using the appropriate primer pairs (Table 1). Finally, the three fragments constituting each gene disruption cassette were stepwise joined by overlap-extension PCR, in each case by using the appropriate external primers. The resulting gene disruption cassette was purified with a PCR purification kit obtained from Macherey-Nagel and used directly to transform S. thermophilus cells by natural transformation.

To construct a T2 derivative with a wild-type $\operatorname{clp} C$ allele, the Up_clpC_F and Down_clpC_R primers were used in PCR to amplify a fragment consisting of the wild-type $c l p C$ gene plus flanking regions of about $1000 \mathrm{bp}$. The resulting PCR fragment was used to transform T2 cells harbouring the pXL plasmid. After overnight growth, 95 single colonies were picked at random from HJGL agar plates and transferred into individual wells of a microtitre plate containing THG medium and D-luciferin (see luciferase reporter assay below). After growth in an Optima FLUOstar luminometer at $37^{\circ} \mathrm{C}$ overnight, one clone was identified that displayed a light emission curve that was different from all the others. Sequence determination of the entire clpC gene of this clone, designated T2-R, revealed that the mutant gene had been replaced by the wild-type allele.

Luciferase reporter assays. Detection of luciferase activity was performed essentially as described by Blomqvist et al. (2006b). Briefly, cultures grown overnight in THG at $37{ }^{\circ} \mathrm{C}$ were diluted to $\mathrm{OD}_{492} 0.05$ in prewarmed THG and further incubated at $37{ }^{\circ} \mathrm{C}$ until they reached $\mathrm{OD}_{492}$ 0.5. Then, cultures were diluted 10 -fold in the same medium and $280 \mu \mathrm{l}$ diluted culture was immediately mixed with firefly Dluciferin $(20 \mu 10 \mathrm{mM}$ D-luciferin solution in THG). If appropriate, STP was added to a final concentration of $250 \mathrm{ng} \mathrm{ml}^{-1}$. Next, samples were transferred into a 96-well Corning NBS plate with a clear bottom. The plate was incubated in an Optima FLUOstar luminometer at $37{ }^{\circ} \mathrm{C}$ for $8 \mathrm{~h}$. Luciferin-luciferase luminescence and $\mathrm{OD}_{492}$ were measured automatically by the luminometer at $10 \mathrm{~min}$ intervals.

Detection of ComX-His by Western analysis. The pXL-His plasmid was electroporated into S. thermophilus LMG 18311 and a $\triangle c l p C$ mutant strain that had been cured from the pXL plasmid. Cultures of these strains were grown overnight at $37{ }^{\circ} \mathrm{C}$ in THG 
Table 1. Primers used in this study

\begin{tabular}{|c|c|c|}
\hline Primer & Gene & Primer sequence $\left(5^{\prime}-3^{\prime}\right)$ \\
\hline Up_clpC_F & $\operatorname{clp} C$ & CAAGCTTTTGACCCССACTA \\
\hline Up_clpC_R & $\operatorname{clp} C$ & CTCGAGGTCGACGGTATCGCGCATCTTCGCCTAAAACAT \\
\hline Down_clpC_F & $c l p C$ & ATTGGATCCATTCCGCGTCAACCGTAAAGCAGGCGTAAAAG \\
\hline Down_clpC_R & $\operatorname{clp} C$ & GAAATCGGAGGCACTGAGAG \\
\hline Up_clpL_F & $c l p L$ & GGTTTATTCTTGTCTAACTCAAAACCA \\
\hline Up_clpL_R & $c l p L$ & CTCGAGGTCGACGGTATCGGACTTTCCTTGGGTGCTACG \\
\hline Down_clpL_F & $c l p L$ & ATTGGATCCATTCCGCGTCAATTGCTCTTGGTAGAAGGCTGA \\
\hline Down_clpL_R & $c l p L$ & AGGTAGCGACCAAACGCTAT \\
\hline Up_clpE_F & $c l p E$ & CCGCCAGGTTATGGAAGTCT \\
\hline Up_clpE_R & $c l p E$ & CTCGAGGTCGACGGTATCGTCCAACATTTGGTGACAAGG \\
\hline Down_clpE_F & $c l p E$ & ATTGGATCCATTCCGCGTCAATTTCTATCTGAGTTGGGAAAGACC \\
\hline Down_clpE_R & $c l p E$ & CCTCTTCGCCCATAGAAACC \\
\hline Up_clpX_F & $\operatorname{clp} X$ & ACGCTTGGTCCTCAATGTTC \\
\hline Up_clpX_R & $\operatorname{clp} X$ & CTCGAGGTCGACGGTATCGGGTGCCATAGAAGGCATGAA \\
\hline Down_clpX_F & $\operatorname{clp} X$ & ATTGGATCCATTCCGCGTCAAACCTGAAGTTGCCCTAGCAG \\
\hline Down_clpX_R & $c l p X$ & GATCCCGTTACTGGCATAGC \\
\hline Up_clpP_F & $c l p P$ & GGCTATGGTTTAGTAACAGGTAA \\
\hline Up_clpP_R & $c l p P$ & AAGCTTAAGATCTAGAGCTCGAACCGGAATCATATGAATTCTCCTT \\
\hline Down_clpP_F & $c l p P$ & AGCATGCATATGCATCCGGAGTGATCAAGCAATAGACAATCTTGTTTTACAT \\
\hline Down_clpP_R & $c l p P$ & CCATTCTGCTCTGACTTACCAT \\
\hline ComX_His_F & $\operatorname{comX}$-His & ATTAGGATCCTTCAAGGTCTAGTCCTCTCTTTTATGACG \\
\hline ComX_His_R & $\operatorname{comX}$-His & AATTAGATATCTCAATGATGATGATGATGATGGTCTTCT TCATTACATGGATCAAAGTC \\
\hline Kana-F & kan & ATCCTCGAGCTCTAGATCTTAAGCTT \\
\hline Kana-R & kan & ACTCCGGATGCATATGCATGCT \\
\hline Spec-F(A) & $\operatorname{spc}$ & CGATACCGTCGACCTCGAG \\
\hline Spec-R & $\operatorname{spc}$ & TTGACGCGGAATGGATCCAAT \\
\hline Up_comX_F & $\operatorname{com} X$ & GACGTAGTAGAGTTGGCGTTCC \\
\hline Up_comX_R & $\operatorname{com} X$ & AAGCTTAAGATCTAGAGCTCGAGGATAAGCGAGATAGAGTCAAAACCA \\
\hline Down_comX_F & $\operatorname{com} X$ & AGCATGCATATGCATCCGGAGTTCCAGTAATTCTTGGAAGGTCAA \\
\hline Down_comX_R & $\operatorname{com} X$ & TCTCCAATTTAATAACAAGAAAAACG \\
\hline
\end{tabular}

supplemented with $2 \mu$ g erythromycin $\mathrm{ml}^{-1}$. The next morning they were diluted to $\mathrm{OD}_{492} 0.05$ in the same medium, immediately induced by adding $250 \mathrm{ng}$ STP ml ${ }^{-1}$ and further incubated at the same temperature in a water bath. When $\mathrm{OD}_{492} 0.2,0.4$ and 0.6 was reached, samples $(10 \mathrm{ml})$ were withdrawn and the bacteria were pelleted by centrifugation. Pellets from these samples were resuspended in 100, 200 and $300 \mu \mathrm{l}$ Laemmli sample buffer, respectively, and stored at $-80{ }^{\circ} \mathrm{C}$. After heating to $95{ }^{\circ} \mathrm{C}$ for $5 \mathrm{~min}$, samples $(35 \mu \mathrm{l})$ were loaded onto $15 \%$ SDS-PAGE precast gels (Bio-Rad), separated and transferred to a PVDF membrane. His-tagged ComX was detected using the Pierce SuperSignal West Pico HisProbe kit according to the manufacturer's instructions.

\section{RESULTS}

\section{Altered transcription of late competence genes in clp-deletion mutants}

In addition to the $c l p P$ gene, the genes encoding the Clp ATP-ases ClpC, ClpE, ClpL and ClpX are present in the genome of $S$. thermophilus. To determine whether any of these Clp proteins are involved in regulating expression of the late competence genes we set out to construct deletion mutants corresponding to each $c l p$ gene. We did not succeed in making a $\Delta c l p X$ mutant, which indicates that
clpX might be an essential gene. Similarly, when trying to knock out the $c l p P$ gene we obtained only a few transformants that grew very poorly. In contrast, $\Delta c l p C, \Delta c l p E$ and $\Delta c l p L$ mutants were readily obtained and displayed growth characteristics similar to those of the wild-type strain. To disrupt the clp genes, we used overlap extension PCR to generate a DNA fragment consisting of an antibiotic selection marker fused to $\sim 1000 \mathrm{bp}$ flanking sequences amplified from the immediate upstream and downstream regions of the genes to be deleted. The resulting fragment was taken up by competent $S$. thermophilus LMG 18311 cells and integrated into the genome of recipients by double-crossover homologous recombination.

Two different methods for inducing the competent state in S. thermophilus were used in this study: a procedure that relies on ectopic expression of ComX from the pXL plasmid (Blomqvist et al., 2006b) and activation of ComX expression by addition of the newly identified $S$. thermophilus competence pheromone ComS (Fontaine et al., 2010). The pXL plasmid, which is a derivative of the pTRKH2 plasmid, contains a $\operatorname{com} X$ gene driven by a bacteriocin promoter and a firefly luciferase gene $(l u c)$ fused to the comEC late gene promoter (Blomqvist et al., 
Table 2. Transformation efficiency of various strains and mutants of $S$. thermophilus

\begin{tabular}{|c|c|c|c|}
\hline Plasmid & Pheromone & Medium & $\begin{array}{c}\text { Transformation } \\
\text { efficiency }^{\star}\end{array}$ \\
\hline \multicolumn{4}{|c|}{ S. thermophilus LMG 18311} \\
\hline pXL & None & THG & 0 \\
\hline pXL & STP & THG & $0.048 \pm 0.006$ \\
\hline None & None & THG & 0 \\
\hline None & Coms & THG & $0.74 \pm 0.05$ \\
\hline None & None & $\mathrm{C}$ & 0 \\
\hline None & ComS & $\mathrm{C}$ & $0.66 \pm 0.1$ \\
\hline \multicolumn{4}{|c|}{ S. thermophilus LMG $18311 \Delta c l p C$} \\
\hline $\mathrm{pXL}$ & None & THG & $0.002 \pm 0.0007$ \\
\hline pXL & STP & THG & $0.046 \pm 0.009$ \\
\hline None & None & THG & 0 \\
\hline None & ComS & THG & $0.05 \pm 0.03$ \\
\hline None & None & $\mathrm{C}$ & $0.02 \pm 0.008$ \\
\hline None & ComS & $\mathrm{C}$ & $0.30 \pm 0.15$ \\
\hline \multicolumn{4}{|c|}{ S. thermophilus LMG 18311-T2 } \\
\hline $\mathrm{pXL}$ & None & THG & $0.089 \pm 0.01$ \\
\hline pXL & STP & THG & $1.75 \pm 0.5$ \\
\hline None & None & THG & 0 \\
\hline None & ComS & THG & $0.074 \pm 0.02$ \\
\hline None & None & C & $0.005 \pm 0.003$ \\
\hline None & ComS & $\mathrm{C}$ & $2.2 \pm 1.0$ \\
\hline \multicolumn{4}{|c|}{ S. thermophilus LMG 18311-T2-R } \\
\hline pXL & None & THG & $2 \times 10^{-4} \pm 3 \times 10^{-5}$ \\
\hline pXL & STP & $\mathrm{THG}$ & $0.071 \pm 0.011$ \\
\hline
\end{tabular}

${ }^{*}$ Transformants (c.f.u.)/total number of bacteria (c.f.u.) $\times 100 \pm$ SEM. Individual transformation experiments were repeated between three and seven times.

2006b). When the peptide pheromone (STP), which controls bacteriocin production in S. thermophilus LMG 18311 , is added to bacteria carrying the pXL plasmid, expression of ComX is induced. Since the bacteriocin promoter is leaky, low level ComX expression can be observed even in the absence of the STP peptide (Blomqvist et al., 2006a, b). As ComX specifically directs transcription from late gene promoters, the intracellular level of this sigma factor is reflected by the level of luciferase activity. To determine whether deletion of $c l p C$, clpE or $c l p L$ affected light production, culture samples of these mutants were grown in 96-well Corning NBS plates at $37^{\circ} \mathrm{C}$ inside an Optima FLUOstar luminometer as described previously (Blomqvist et al., 2006b). The results showed that an uninduced pXL strain lacking $c l p C$ produced much more light than the corresponding uninduced wild-type strain (Fig. 1a). Intriguingly, STPinduced and uninduced cultures of this $c l p C$ mutant displayed identical levels of luciferase activity. Deletion of the $c l p E$ gene resulted in increased reporter activity in both induced and uninduced cultures, whereas the level of light production in a $\Delta c l p L$ mutant remained largely unaltered compared with the wild-type (Fig. 1b). As deletion of $c l p C$ resulted in a dramatic increase in light emission in uninduced cultures, i.e. under conditions where the intracellular levels of ComX are low, we decided to focus on $\mathrm{ClpC}$ and its role in the regulation of natural transformation in S. thermophilus LMG 18311.

\section{Deletion of $c / p C$ strongly upregulates transcription of late competence genes without affecting the level of comX transcription}

The strong increase in late gene transcription observed in a $\triangle c l p C$ mutant indicates that $\mathrm{ClpC}$ directly or indirectly influences the level or activity of ComX in the cell. To further elucidate these matters we took advantage of two previously constructed reporter plasmids (Blomqvist et al., 2006b). In one of these, pXP, the luciferase gene is driven by the $\operatorname{com} X$ promoter, while in the other, pEAP, the ComEC late gene promoter is placed upstream of the $l u c$ reporter (Blomqvist et al., 2006b). None of these plasmids contains the STP-inducible comX gene carried on the pXL plasmid described above. When the pXP plasmid was introduced into $S$. thermophilus wild-type cells and a $\Delta c l p C$ mutant by electroporation, no difference in reporter activity was observed between the two strains (Fig. 1d). These results show that $\mathrm{ClpC}$ does not affect the level of ComX expression in S. thermophilus cells growing in THG. Interestingly, however, when the pEAP plasmid was introduced into wild-type and $\triangle c l p C$ cells, light was produced only by the cells lacking ClpC (Fig. 1c). To make sure that the emitted light results from ComX-driven transcription of the pEAP-located luciferase gene, the $\operatorname{comX}$ gene was deleted from the chromosome of $\Delta c l p C$ cells carrying the pEAP plasmid. Luciferase reporter assays showed that no light was produced by the resulting $\operatorname{com} X-$ deficient strain (results not shown). This proves that $\mathrm{ClpC}$ acts through ComX and not through another regulatory protein that in theory might have affected transcription of the late competence genes. Together, these results indicate that ClpC regulates the level or activity of ComX post-translationally.

\section{The clpC deletion mutant develops the competent state spontaneously when grown in $\mathbf{C}$ medium}

To determine whether the increased level of late gene expression observed in the $\triangle c l p C$ mutant has a positive effect on the transformability of this mutant compared with the wild-type strain, a number of transformation assays were performed (see Table 2). A pCR 2.1-TOPO plasmid carrying a kanamycin resistance gene flanked at both ends by about $1000 \mathrm{bp}$ DNA originating from the upstream and downstream regions of the S. thermophilus comEC gene was used as donor DNA in these transformation experiments. In THG medium, assays carried out with $\Delta c l p C$ and wild-type strains carrying the pXL plasmid demonstrated that the $\Delta c l p C$ strain transformed with the same efficiency as the corresponding wild-type strain when the strains were induced by STP. However, in contrast with 

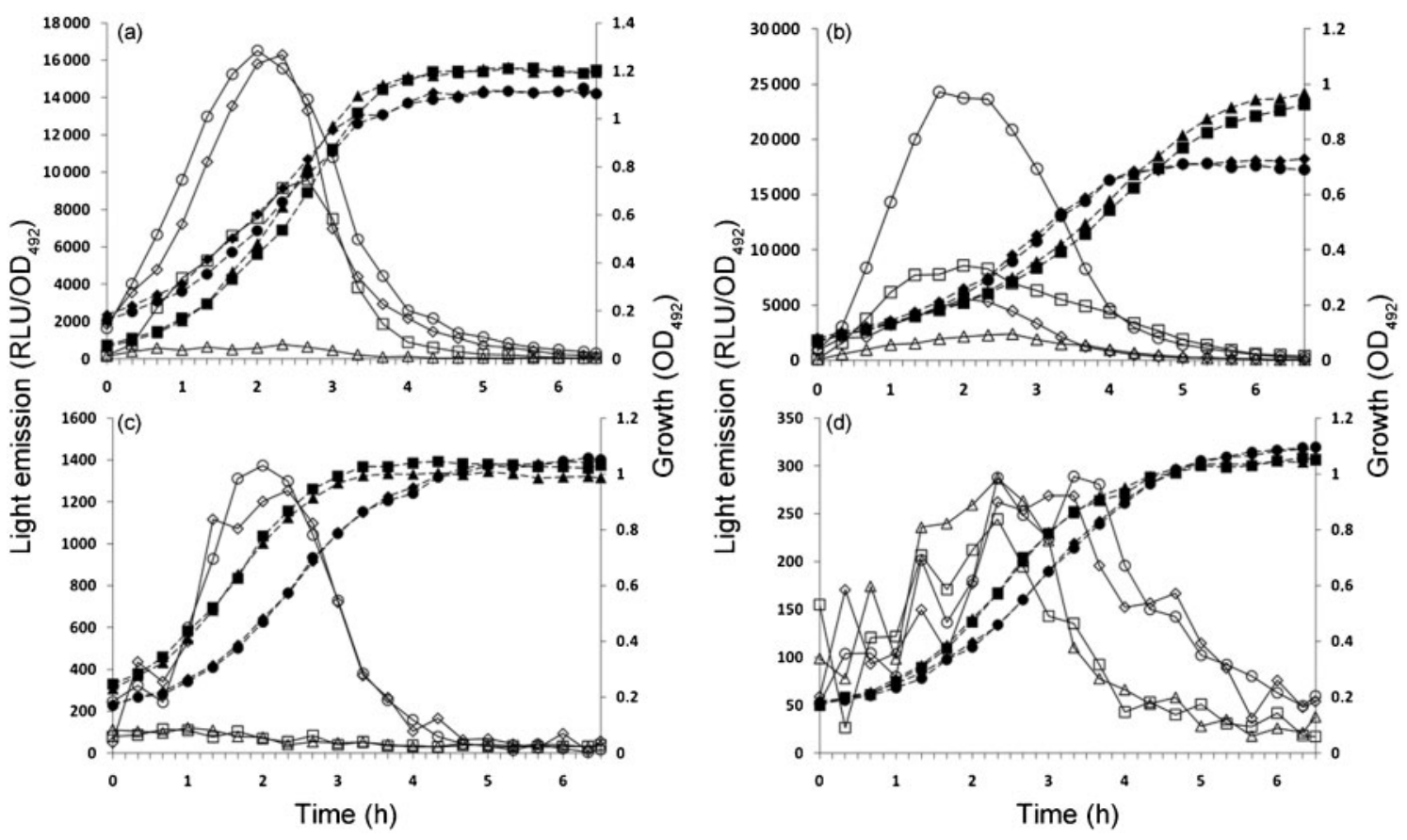

Fig. 1. Luciferase reporter assays that show the activity of the competence-specific promoters $\mathrm{P}_{\text {comEC }}$ and $\mathrm{P}_{\text {comX }}$ in wild-type (WT) and clp deletion mutants of $S$. thermophilus. Continuous lines and open symbols represent light emission [relative light units (RLU) divided by $\mathrm{OD}_{492}$ ], while dashed lines and filled symbols represent growth curves. Cultures treated with STP received $250 \mathrm{ng} \mathrm{ml}^{-1}$ at time zero. (a) WT (squares, +STP; triangles, -STP) and $\Delta c / p C$ mutant (circles, +STP; diamonds, -STP) carrying the $\mathrm{PXL}$ plasmid. $\mathrm{pXL}$ contains a comX gene driven by a leaky bacteriocin promoter that is inducible by the STP peptide pheromone. In addition the $\mathrm{pXL}$ plasmid contains a firefly luciferase gene (luc) fused to the comEC late gene promoter. (b) $\Delta c / p L$ mutant (squares, +STP; triangles, $-\mathrm{STP}$ ) and $\Delta c / p E$ mutant (circles, +STP; diamonds, $-\mathrm{STP}$ ) carrying the $\mathrm{pXL}$ plasmid. (c) WT (squares, +STP; triangles, -STP) and $\Delta c / p C$ mutant (circles, +STP; diamonds, - STP) carrying the pEAP plasmid. The pEAP plasmid differs from the pXL plasmid in that it contains only a luc gene fused to the comEC late gene promoter. (d) WT (squares, +STP; triangles, -STP) and $\Delta c / p C$ mutant (circles, +STP; diamonds, $-\mathrm{STP}$ ) carrying the pXP plasmid. The pXP plasmid contains a luc gene fused to the $\operatorname{com} X$ promoter. These experiments were repeated at least three times with highly similar results.

the wild-type strain, the $\Delta c l p C$ strain was also naturally slightly transformable without addition of STP, i.e. without STP-induced overexpression of ComX. This is due to the leaky bacteriocin promoter situated upstream of $\operatorname{com} X$, which allows low-level ComX expression even in the absence of STP. In wild-type and $\triangle c l p C$ strains lacking the pXL plasmid, transformation in THG medium was only observed in the presence of the ComS pheromone. No spontaneous transformation was detected under these conditions. Interestingly, however, a screening of different growth media revealed that a $\Delta c l p C$ mutant lacking the pXL plasmid became spontaneously competent for natural transformation when grown in semi-synthetic $\mathrm{C}$ medium. No transformants were obtained when the corresponding wild-type strain was grown in this medium (Table 2).

\section{Identification of a competence-up mutant}

When testing different clones of the $\Delta c l p E$ and $\Delta c l p L$ mutants described above we noticed that in both cases two different phenotypes appeared. One variant corresponded to the $\Delta c l p E$ and $\Delta c l p L$ phenotypes described in Fig. 1(b), while the other was more similar to the $\Delta c l p C$ phenotype (Fig. 1a). The characteristic phenotype of $\Delta c l p C$ mutants containing the pXL plasmid is that there is no difference between the amount of light produced in the presence or absence of STP induction. The solution to this puzzle turned out to be that our stock of the S. thermophilus LMG 18311 strain was not homogeneous, but contained two variants with respect to transformability. In the presence of the pXL plasmid, the majority of the cells representing the wild-type (WT) transformed with significantly less efficiency than the other variant (T2). The two different phenotypes, WT and T2, were stable and did not change even after cultivation for hundreds of generations. All experiments presented in Fig. 1 are performed with WT or clp mutants of WT cells. As the phenotype of T2 cells closely resembled that of WT cells lacking a functional $\operatorname{clp} C$ gene (Fig. 2), we speculated that T2 cells might represent a natural $c l p C$ mutant. We therefore amplified the $c l p C$ gene from T2 cells by PCR and sequenced the resulting DNA fragment. Indeed, the results showed that the $\mathrm{T} 2 \mathrm{clpC}$ gene 

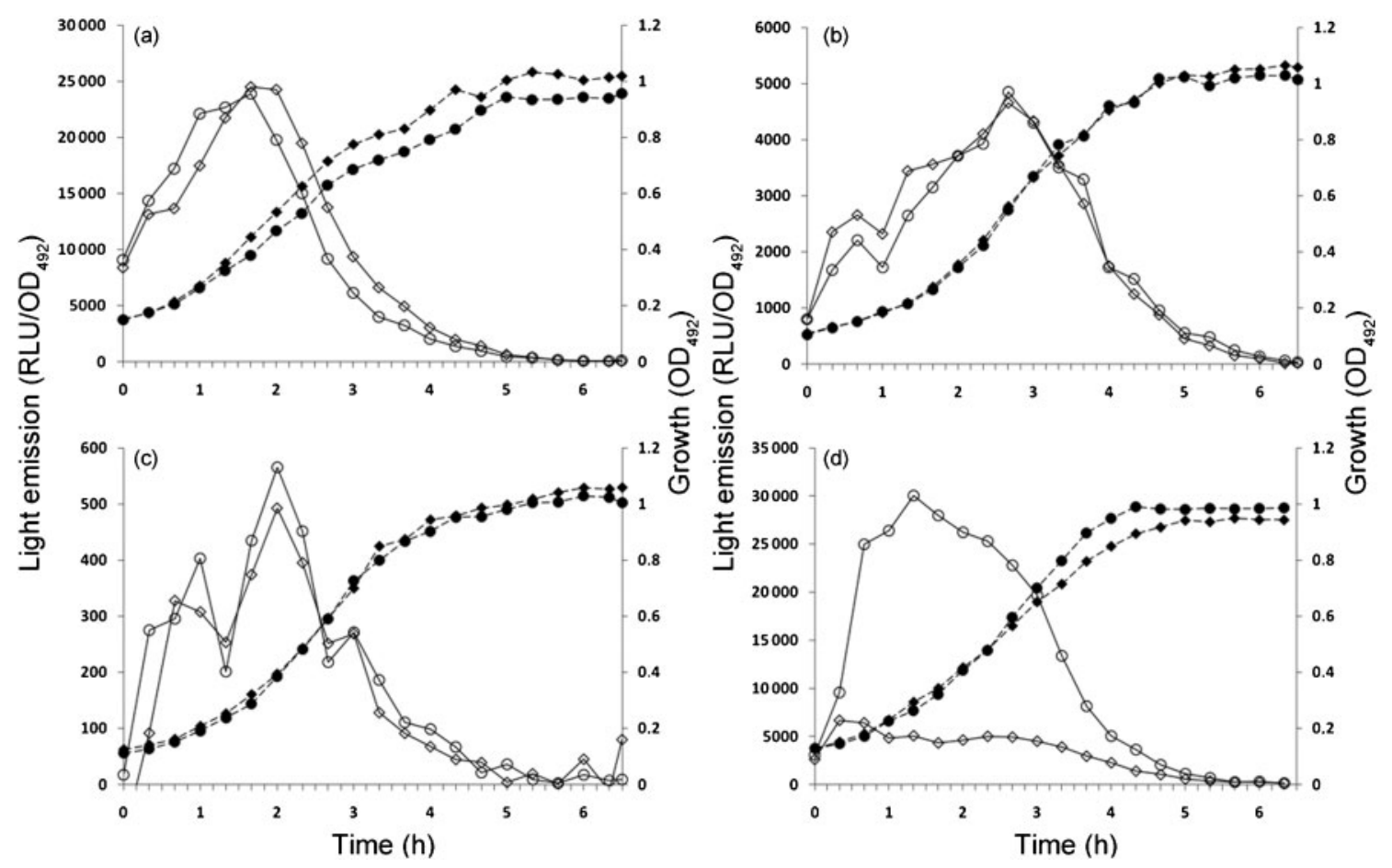

Fig. 2. Luciferase reporter assays that show the activity of the competence-specific promoters $P_{\text {comEC }}$ and $P_{\text {com } X}$ in a natural isolate of $S$. thermophilus termed T2. Continuous lines and open symbols represent light emission (RLU divided by $\left.\mathrm{OD}_{492}\right)$, while dashed lines and filled symbols represent growth curves. Cultures treated with STP received $250 \mathrm{ng} \mathrm{ml}^{-1}$ at time zero. (a) T2 strain (circles, +STP; diamonds, -STP) carrying the pXL plasmid. pXL contains a comX gene driven by a leaky bacteriocin promoter that is inducible by the STP peptide pheromone. In addition the $\mathrm{pXL}$ plasmid contains a firefly luciferase gene (/uc) fused to the comEC late gene promoter. (b) T2 strain (circles, +STP; diamonds, -STP) carrying the pEAP plasmid. The pEAP plasmid differs from the $\mathrm{PXL}$ plasmid in that it contains only a luc gene fused to the comEC late gene promoter. (c) T2 strain (circles, +STP; diamonds, -STP) carrying the pXP plasmid. The pXP plasmid contains a luc gene fused to the com $X$ promoter. (d) T2-R strain (circles, +STP; diamonds, -STP). This strain is identical to the T2 strain except that it carries a repaired $c / p C$ gene. In the T2-R strain the $\mathrm{G}_{1630} \rightarrow \mathrm{T}_{1630}$ nonsense mutation identified in the clpC gene of the T2 strain has been backmutated to the wild-type sequence. These experiments were repeated at least three times with highly similar results.

contains a point mutation $\left(\mathrm{G}_{1630} \rightarrow \mathrm{T}_{1630}\right)$ resulting in the introduction of a stop codon at amino acid position 544 . Consequently, the $\operatorname{clp} C$ gene product of the T2 strain will be shortened by 273 aa at the C-terminal end. The $\mathrm{G}_{1630} \rightarrow \mathrm{T}_{1630}$ point mutation was not introduced by the PCR used to generate the sequencing template, as the cplC gene was amplified from the T2 strain and sequenced several times with the same result. To determine whether the $G_{1630} \rightarrow T_{1630}$ point mutation is responsible for the particular phenotype of the $\mathrm{T} 2$ strain, we reintroduced a functional $c l p C$ gene into this strain by transformation with a PCR fragment consisting of the wild-type allele plus flanking regions of about $1000 \mathrm{bp}$. The resulting strain was termed T2-R. Interestingly, luciferase reporter assays carried out with the T2-R strain showed that it behaved differently from its parental strain (Fig. 1a, d). When induced with the STP pheromone, T2-R emitted roughly the same amount of light as the T2 strain. However, in uninduced cells, where ComX is expressed only at low levels, light emission in the T2-R cells was reduced about fivefold compared with the T2 cells. This result demonstrates that the $\mathrm{G}_{1630} \rightarrow \mathrm{T}_{1630}$ nonsense mutation strongly contributes to the high-level late gene expression observed in uninduced T2 cells. Compared with wild-type cells harbouring the pXL plasmid, the uninduced T2-R strain still produces at least fivefold more light (see Figs $1 \mathrm{a}$ and $2 \mathrm{~d})$. This indicates that the $\mathrm{T} 2$ strain carries additional mutations that together with the $\mathrm{G}_{1630} \rightarrow \mathrm{T}_{1630}$ point mutation give rise to the $\mathrm{T} 2$ phenotype.

Estimation of the transformation efficiencies of the WT $\Delta c l p C$ mutant and the T2 strain showed that the transformation rate of the T2 strain is about 40 -fold higher than that of the $\Delta c l p C$ mutant when the strains are grown in plain THG medium and THG containing the STP pheromone. In THG medium supplemented with the ComS pheromone their transformation rates are similar, while the $\mathrm{T} 2$ strain transformed about sevenfold better in $\mathrm{C}$ medium containing ComS. However, when grown in $\mathrm{C}$ medium in the absence of ComS the $\Delta c l p C$ mutant gives 
rise to about four times more transformants than the T2 strain (Table 2). Comparison of the transformabilities of the T2 and T2-R strains shows that the T2 strain transforms about 25 -fold better than the T2-R strain. This result demonstrates that the high transformation efficiency displayed by the T2 strain is to a large extent due to the nonsense mutation that disrupts the function of its $c l p C$ gene. Compared with the WT $\Delta c l p C$ mutant, the transformation rate of the T2-R strain is slightly higher when the strains are grown in THG and induced to competence by the STP pheromone. In the absence of the STP pheromone, i.e. in uninduced cultures, no kanamycinresistant mutants were ever obtained with the WT $\Delta c l p C$ mutant. In contrast, a low number of transformants was consistently obtained with the T2-R strain (Table 2). Consequently, it appears that the $\mathrm{T} 2$ strain has acquired mutations in addition to the $\mathrm{G}_{1630} \rightarrow \mathrm{T}_{1630}$ nonsense mutation that contribute to its enhanced transformability compared with the $\Delta c l p C$ mutant.

\section{ClpC affects ComX accumulation in STP-induced cells}

To investigate whether $\mathrm{ClpC}$ mediates proteolytic degradation of ComX we exchanged the $\operatorname{com} X$ gene in the $\mathrm{pXL}$ plasmid with a His-tagged version. The $6 \times$ His tag was inserted at the C-terminal end of ComX. The resulting pXL-His plasmid was introduced into the WT and $\Delta c l p C$ strains by electroporation giving rise to the WT-His and $\Delta c l p C$-His strains, respectively. Since the $\Delta c l p C$ strain originally contained the pXL plasmid, it had to be cured of this plasmid before introduction of pXL-His. To compare the levels of ComX in the WT-His and $\Delta c l p C$-His strains, samples from STP-induced cultures were collected at different time points and analysed by Western blotting. Overnight cultures were diluted in fresh prewarmed THG and cultivated at $37{ }^{\circ} \mathrm{C}$ until they reached $\mathrm{OD}_{492} 0.05$. At

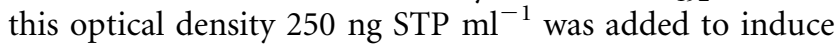
ectopic expression of the ComX-His protein. After further incubation at $37^{\circ} \mathrm{C}$, samples for Western analysis were withdrawn from WT-His and $\Delta c l p C$-His cultures when they reached $\mathrm{OD}_{492} 0.2,0.4$ and 0.6 . The results showed that $\mathrm{ClpC}$ mediates proteolysis of ComX during the time period immediately following STP induction, i.e. until the cultures reached $\mathrm{OD}_{492}$ 0.2. However, 1.5 generations later the picture was radically changed. In samples collected from cultures at $\mathrm{OD}_{492}$ 0.6, ComX had accumulated to high levels in WT cells, while its concentration in $\Delta c l p C$ cells was strongly reduced (Fig. 3).

\section{DISCUSSION}

Our results show that under some conditions, ClpC mutants display increased expression of late competence genes and higher transformation efficiency than the corresponding WT strain. The effect of ClpC is best seen when the level of ComX expression is low, such as in the

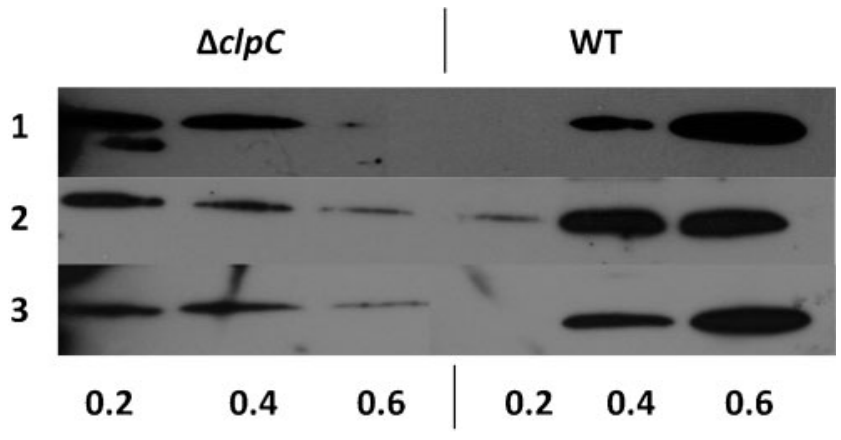

Fig. 3. Western blot analyses comparing levels of ComX-His expression in $S$. thermophilus wild-type (WT) and $\Delta c / p C$ strains grown at $37{ }^{\circ} \mathrm{C}$ in THG medium. Both strains carry the pXL-His plasmid. ComX-His expression was induced by addition of STP $\left(250 \mathrm{ng} \mathrm{ml}^{-1}\right.$ ) at $\mathrm{OD}_{492}$ 0.05. Samples for Western analysis were collected when cultures reached $\mathrm{OD}_{492} 0.2,0.4$ and 0.6 . The results of three independent experiments are shown.

uninduced $\Delta c l p C$ mutant carrying the pXL plasmid (Fig. 1a and Table 2). In this strain, the leaky bacteriocin promoter controlling transcription of $\operatorname{comX}$ allows production of small amounts of ComX in the absence of the STP inducer. Luciferase reporter assays carried out with uninduced $\triangle c l p C$ and WT cells, both containing the pXL plasmid, revealed a huge difference in late gene expression (Fig. 1a). Since deletion of the $c l p C$ gene does not stimulate the rate of $\operatorname{com} X$ transcription (Fig. 1d), we concluded that the high $l u c$ activity exhibited by the uninduced $\Delta c l p C$ strain is most likely due to increased stability of the ComX protein.

To obtain additional evidence for this hypothesis we constructed the plasmid pXL-His, which encodes a Histagged version of ComX driven by an STP-inducible bacteriocin promoter. When WT and $\triangle c l p C$ bacteria carrying the pXL-His plasmid reached $\mathrm{OD}_{492} 0.05$, STP was added to induce expression of ComX. Samples were collected at $\mathrm{OD}_{492} \quad 0.2,0.4$ and 0.6 and subjected to Western analysis. Interestingly, ComX-His was undetectable in two of three independent Western analyses carried out with WT bacteria harvested at $\mathrm{OD}_{492}$ 0.2, i.e. two generations after the STP pheromone was added (Fig. 3). In the third experiment, ComX-His was detected, but the band was very weak. In contrast, strong bands representing ComX-His were obtained with the $\Delta c l p C$ mutant (Fig. 3). These data show that ClpC mediates degradation of ComX at low cell densities in the period immediately following STP induction. This is in agreement with the results of the reporter assay experiments presented in Fig. 1(a). Induction of ComX expression in these experiments was performed exactly as in the Western analyses, i.e. by addition of STP at $\mathrm{OD}_{492} 0.05$. When the experiment was carried out like this, ComX in a $\Delta c l p C$ mutant is not degraded by the ClpCP complex during a period corresponding to about two generations post-induction (Fig. 3). As a consequence, the concentration of ComX required to 
elicit strong transcriptional activation of the late competence genes is reached in both induced and uninduced cells, even though uninduced cells produce much less ComX than the corresponding STP-treated cells. The results displayed in Fig. 1(a) also show that light emission peaks earlier in $\triangle c l p C$ than WT cultures. The reason for this is that ComX is not degraded in $\Delta c l p C$ cultures in the period following STP induction and therefore accumulates and triggers late gene expression more rapidly.

ClpC-mediated degradation of ComX vanished at higher ODs, i.e. about three generations after STP induction. The results depicted in Fig. 3 clearly show that the level of ComX-His increases with time and cell density in WT cells, while the $\triangle c l p C$ mutant cells behave in the opposite manner displaying very low ComX levels at $\mathrm{OD}_{492}$ 0.6. The increasing amounts of ComX-His observed in WT cells after STP induction presumably results from the combined effects of continuous high expression of ComX-His and saturation of ClpC. However, the existence of additional mechanisms controlling the levels and activity of this sigma factor cannot be ruled out. The virtual disappearance of ComX-His in $\Delta c l p C$ cells at $\mathrm{OD}_{492} 0.6$ was totally unexpected. In theory, it might be caused by ComX-His degradation, shutdown of ComX-His synthesis, or a combination of the two. The STP peptide pheromone used to induce ComX synthesis, concomitantly induces transcription of genes involved in bacteriocin production in $S$. thermophilus. It is therefore conceivable that the increase in bacteriocin-related protein synthesis resulting from the use of STP to some degree affects the kinetics of ComX degradation by the Clp proteolytic complexes and that the effect is not the same in WT and $\Delta c l p C$ cells. More research is required to elucidate these matters.

Previous studies have shown that viable deletion mutants of $c l p C, c l p E, c l p L$ and $c l p P$ can be generated in $S$. pneumoniae. Only one Clp protein, $\mathrm{ClpX}$, was found to be essential in the pneumococcus (Robertson et al., 2003; Piotrowski et al., 2009b). As we were unsuccessful in generating a $c l p X$ mutant in $S$. thermophilus, it is reasonable to assume that ClpX is essential also in this species. In $S$. pneumoniae it was demonstrated that the requirement for $c l p X$ is relieved by deletion of spr1630, a gene of unknown function (Piotrowski et al., 2009b). A corresponding mechanism cannot account for our results, however, since the spr1630 gene is missing from the genome of $S$. thermophilus LMG 18311.

Characterization of pneumococcal strains with non-functional $\operatorname{clpP}$ genes revealed that these mutants displayed increased expression of early and late competence genes, most likely because Clp proteases inhibit expression of the comCDE operon under conditions inappropriate for competence development (Chastanet et al., 2001; Robertson et al., 2002). A similar observation has been made for Streptococcus pyogenes (Opdyke et al., 2003). Recently, deletion of the chaperone members of the Clp family demonstrated that ClpE affects the stability of ComX in S. pneumoniae.
Piotrowski et al. (2009a) found that the ClpEP complex contributes to the degradation of the ComX protein in the period following competence induction. The competent state only lasts for about $40 \mathrm{~min}$, after which it is shut down by an unknown mechanism (Håvarstein et al., 1995). It has therefore been speculated that ClpEP degradation of ComX might be responsible for the cessation of competence in $S$. pneumoniae. This turned out not to be the case, however, as transcription of the late genes and consequently the competent state, terminates despite persistently high levels of ComX in a $\Delta c l p P$ mutant (Piotrowski et al., 2009a). Interestingly, our results show that deletion of the $c l p E$ gene in $S$. thermophilus causes significantly increased late gene expression (Fig. 1b). It is therefore possible that ClpE serves similar functions in S. thermophilus and S. pneumoniae with respect to competence regulation.

The Clp ATPase, ClpL, which appears to be present only in Gram-positive bacteria, is involved in tolerance to temperature stress in S. thermophilus. Varcamonti et al. (2006) showed that expression of the ClpL protein increases in cells subjected to heat and cold shock. $S$. thermophilus cells lacking a functional clpL gene grew significantly slower at $20{ }^{\circ} \mathrm{C}$ and were much less able to tolerate exposure to $60{ }^{\circ} \mathrm{C}$. The observed thermosensitivity of the clpL mutant suggests that ClpL functions as a chaperone that assists in protein folding. ClpL lacks the tripeptide ClpP recognition sequence (IGF loop) and is therefore not able to form a proteolytic complex with $\mathrm{ClpP}$ (Frees et al., 2007). Consequently, ClpL is presumably not involved in controlling the stability and activity of transcriptional regulators. Our finding that disruption of the $c l p L$ gene does not affect expression of the late competence genes in STP-induced cells (Fig. 1b) is in accordance with the view that ClpL mediates protein folding rather than protein degradation.

In contrast with S. pneumoniae, where inactivation of $c l p C$ does not significantly affect expression of early and late competence genes (Chastanet et al., 2001), ClpC clearly has a strong effect on the stability of the ComX protein in $S$. thermophilus. Together our results indicate that the biological role of $\mathrm{ClpC}$ is to suppress expression of the late competence genes under conditions that are suboptimal for competence development. If left unchecked, it is likely that low-level activation or just transcriptional noise could cause gradual accumulation of intracellular ComX, leading to development of the competent state at an inappropriate time and place. Apparently, ClpC prevents this from happening by degrading ComX. However, under conditions that strongly favour competence development in S. thermophilus, $\mathrm{ClpC}$ is presumably quickly saturated by high levels of ComX and is therefore not able to block late gene expression and development of the competent state.

The competence-up mutant, termed T2, turned out to be a natural $\Delta c l p C$ mutant. Our results show that the T2 strain transforms considerably better than the $S$. thermophilus LMG 18311 wild-type strain under most conditions tested 
(Table 2). Considering that deletion of the $\operatorname{clp} C$ gene has a strong positive effect on luc expression in uninduced WT cells carrying the pXL plasmid (Fig. 1a), it is reasonable to assume that the T2 phenotype results from a nonfunctional $c l p C$ gene. To further investigate this, the $\mathrm{G}_{1630} \rightarrow \mathrm{T}_{1630}$ nonsense mutation was back-mutated to the wild-type sequence, giving rise to the $\mathrm{T} 2-\mathrm{R}$ strain. The transformation efficiency of T2-R strain dropped 25-fold compared with the T2 strain (Table 2). Similarly, the luc expression in uninduced cells dropped about fivefold compared with the T2 strain (Fig. 2a, d). These results confirm that the T2 phenotype is predominantly the result of the $\mathrm{G}_{1630} \rightarrow \mathrm{T}_{1630}$ nonsense mutation. However, there might be additional changes in the $\mathrm{T} 2$ genome that contribute to its elevated transformation rate. This is most clearly indicated in the luciferase reporter assays presented in Figs 1(a) and 2(d). Light emission is at least fivefold higher in the uninduced T2-R strain compared with the uninduced WT strain, demonstrating that low levels of ComX activate late gene transcription more efficiently in T2-R than in WT cells. With respect to transformation efficiency, the difference between the T2-R and WT strains is relatively small. In THG medium, STP-induced T2-R cells transform only slightly better than WT cells treated the same way. However, a clear difference was observed between uninduced cultures of the two strains, i.e. at conditions where the intracellular concentration of ComX is low. A few transformants were consistently obtained with the T2-R strain, but no transformants were ever observed when $\mathrm{Kan}^{\mathrm{R}}$-DNA was added to the WT strain (Table 2). In sum, our data show that although the T2 phenotype is largely caused by the $G_{1630} \rightarrow T_{1630}$ nonsense mutation, the T2 strain probably contains additional mutations that further increase its transformability.

\section{ACKNOWLEDGEMENTS}

This work was supported by The Research Council of Norway.

\section{REFERENCES}

Blomqvist, T., Steinmoen, H. \& Håvarstein, L. S. (2006a). Pheromone-induced expression of recombinant proteins in Streptococcus thermophilus. Arch Microbiol 186, 465-473.

Blomqvist, T., Steinmoen, H. \& Håvarstein, L. S. (2006b). Natural genetic transformation: A novel tool for efficient genetic engineering of the dairy bacterium Streptococcus thermophilus. Appl Environ Microbiol 72, 6751-6756.

Chastanet, A., Prudhomme, M., Claverys, J. P. \& Msadek, T. (2001). Regulation of Streptococcus pneumoniae clp genes and their role in competence development and stress survival. J Bacteriol 183, 7295-7307.

Fontaine, L., Boutry, C., Guédon, E., Guillot, A., Ibrahim, M., Grossiord, B. \& Hols, P. (2007). Quorum-sensing regulation of the production of Blp bacteriocins in Streptococcus thermophilus. J Bacteriol 189, 7195-7205.

Fontaine, L., Boutry, C., de Frahan, M. H., Delplace, B., Fremaux, C., Horvath, P., Boyaval, P. \& Hols, P. (2010). A novel pheromone quorum-sensing system controls the development of natural com- petence in Streptococcus thermophilus and Streptococcus salivarius. J Bacteriol 192, 1444-1454.

Frees, D., Savijoki, K., Varmanen, P. \& Ingmer, H. (2007). Clp ATPases and ClpP proteolytic complexes regulate vital biological processes in low GC, Gram-positive bacteria. Mol Microbiol 63, 1285-1295.

Gardan, R., Besset, C., Guillot, A., Gitton, C. \& Monnet, V. (2009). The oligopeptide transport system is essential for the development of natural competence in Streptococcus thermophilus strain LMD-9. J Bacteriol 191, 4647-4655.

Håvarstein, L. S., Coomaraswamy, G. \& Morrison, D. A. (1995). An unmodified heptadecapeptide pheromone induces competence for genetic transformation in Streptococcus pneumoniae. Proc Natl Acad Sci U S A 92, 11140-11144.

Johnsborg, O. \& Håvarstein, L. S. (2009). Regulation of natural genetic transformation and acquisition of transforming DNA in Streptococcus pneumoniae. FEMS Microbiol Rev 33, 627-642.

Kress, W., Maglica, Z. \& Weber-Ban, E. (2009). Clp chaperoneproteases: structure and function. Res Microbiol 160, 618-628.

Lacks, S. A. \& Hotchkiss, R. D. (1960). A study of the genetic material determining an enzyme in Pneumococcus. Biochim Biophys Acta 39, 508-518.

Lee, M. S. \& Morrison, D. A. (1999). Identification of a new regulator in Streptococcus pneumoniae linking quorum sensing to competence for genetic transformation. J Bacteriol 181, 5004-5016.

Martin, B., Prudhomme, M., Alloing, G., Granadel, C. \& Claverys, J. P. (2000). Cross-regulation of competence pheromone production and export in the early control of transformation in Streptococcus pneumoniae. Mol Microbiol 38, 867-878.

Opdyke, J. A., Scott, J. R. \& Moran, C. P., Jr (2003). Expression of the secondary sigma factor $\sigma^{\mathrm{X}}$ in Streptococcus pyogenes is restricted at two levels. J Bacteriol 185, 4291-4297.

Pestova, E. V., Håvarstein, L. S. \& Morrison, D. A. (1996). Regulation of competence for genetic transformation in Streptococcus pneumoniae by an auto-induced peptide pheromone and a two-component regulatory system. Mol Microbiol 21, 853-862.

Piotrowski, A., Luo, P. \& Morrison, D. A. (2009a). Competence for genetic transformation in Streptococcus pneumoniae: termination of activity of the alternative sigma factor ComX is independent of proteolysis of ComX and ComW. J Bacteriol 191, 3359-3366.

Piotrowski, A., Burghout, P. \& Morrison, D. A. (2009b). spr1630 is responsible for the lethality of $c l p X$ mutations in Streptococcus pneumoniae. J Bacteriol 191, 4888-4895.

Podbielski, A., Spellerberg, B., Woischnik, M., Pohl, B. \& Lütticken, R. (1996). Novel series of plasmid vectors for gene inactivation and expression analysis in group A streptococci (GAS). Gene 177, 137-147.

Robertson, G. T., Ng, W. L., Foley, J., Gilmour, R. \& Winkler, M. E. (2002). Global transcriptional analysis of $c l p P$ mutations of type 2 Streptococcus pneumoniae and their effects on physiology and virulence. J Bacteriol 184, 3508-3520.

Robertson, G. T., Ng, W. L., Gilmour, R. \& Winkler, M. E. (2003). Essentiality of $c l p X$, but not $c l p P, c l p L, c l p C$, or $c l p E$, in Streptococcus pneumoniae R6. J Bacteriol 185, 2961-2966.

Sung, C. K. \& Morrison, D. A. (2005). Two distinct functions of ComW in stabilization and activation of the alternative sigma factor ComX in Streptococcus pneumoniae. J Bacteriol 187, 3052-3061.

Varcamonti, M., Arsenijevic, S., Martirani, L., Fusco, D., Naclerio, G. \& De Felice, M. (2006). Expression of the heat shock gene $c l p L$ of Streptococcus thermophilus is induced by both heat and cold shock. Microb Cell Fact 5, 6.

Edited by: M. Kilian 